\title{
Synthesis of pan-JAK Inhibitor PF-06263276
}

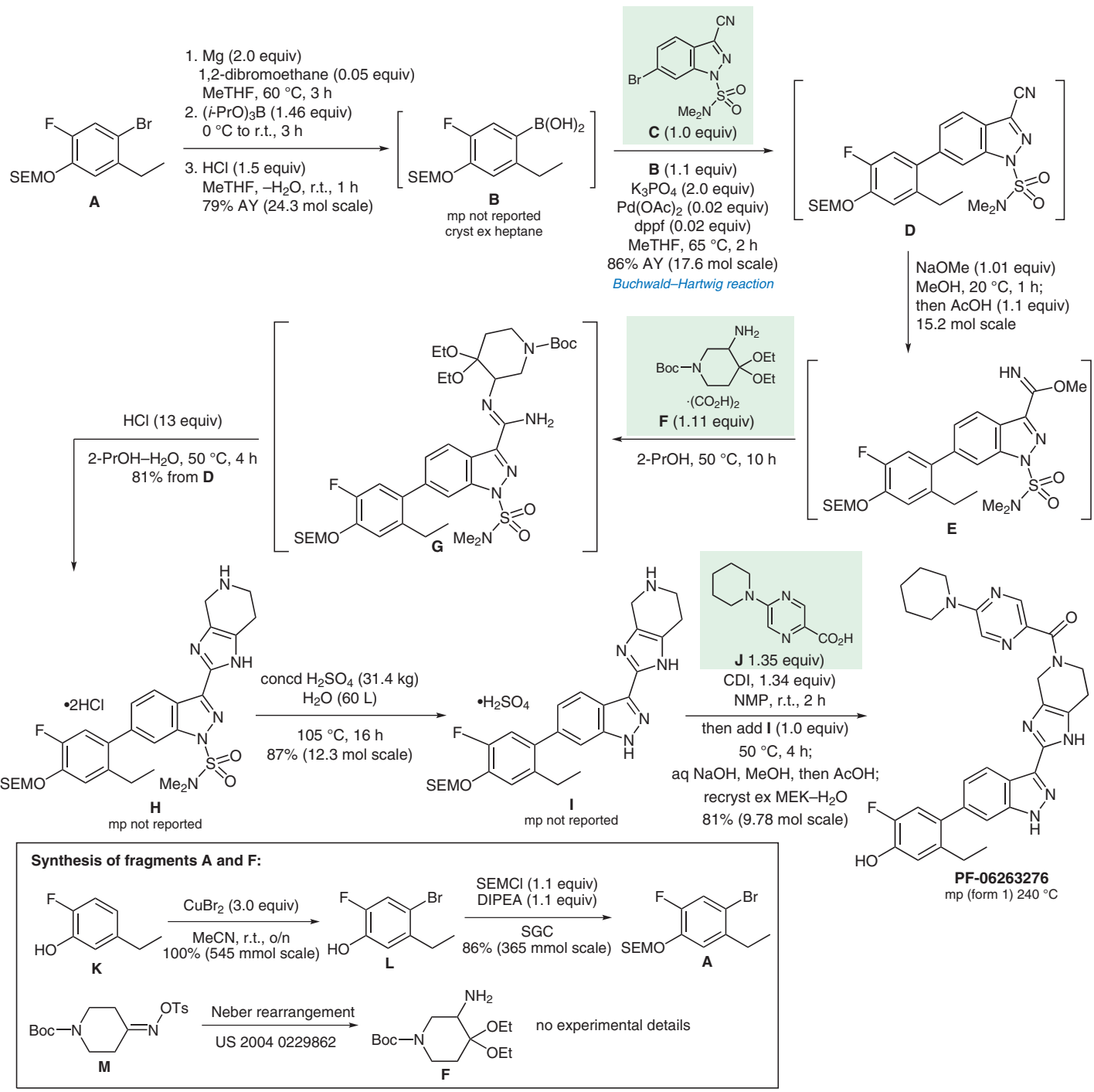

Synthesis of Natural

Products and

Potential Drugs

PF-06263276

Suzuki-Miyaura cross-coupling

imidazole ring

formation

amidation

dimethylsulfamoyl protecting group

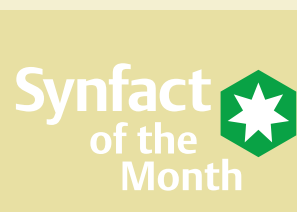

Significance: PF-06263276 is a pan-Janus kinase inhibitor that is of interest for the treatment of inflammatory diseases. The synthesis depicted is a telescoped variant of the discovery synthesis ( $P$. Jones et al. J. Med. Chem. 2017, 60, 767). A notable feature is the three-step conversion of nitrile $\mathbf{D}$ into crystalline imidazole dihydrochloride $\mathbf{H}$ in $81 \%$ yield.
Comment: The dimethylsulfamoyl protecting group on indazole D served two purposes. It enhanced the electrophilicity of the nitrile group, and it increased the stability of the imidate $\mathbf{E}$. Unfortunately, hydrolysis of the dimethylsulfamoyl group required $35 \%$ aqueous sulfuric acid at $105^{\circ} \mathrm{C}$ for sixteen hours. 